\title{
Norois
}

Environnement, aménagement, société

\section{La « zone à défendre » de Notre-Dame-des-Landes ou l'habiter comme politique}

The "Area to Be Defended" at Notre-Dame-des-Landes, or the Art of Inhabiting Places as Political Strategy

\section{Frédéric Barbe}

\section{(2) OpenEdition}

\section{Journals}

Édition électronique

URL : http://journals.openedition.org/norois/5898

DOI : $10.4000 /$ norois. 5898

ISBN : 978-2-7535-5227-2

ISSN : $1760-8546$

\section{Éditeur}

Presses universitaires de Rennes

\section{Édition imprimée}

Date de publication : 17 octobre 2016

Pagination : 109-130

ISBN : 978-2-7535-5222-7

ISSN : 0029-182X

\section{Référence électronique}

Frédéric Barbe, «La "zone à défendre » de Notre-Dame-des-Landes ou l'habiter comme politique », Norois [En ligne], 238-239 | 2016, mis en ligne le 17 octobre 2018, consulté le 01 mai 2019. URL : http://journals.openedition.org/norois/5898; DOI : 10.4000/norois.5898 


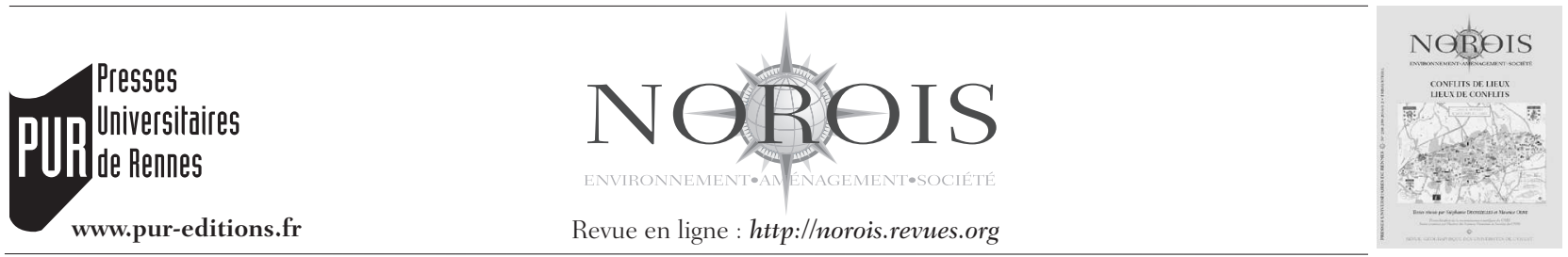

\title{
La «zone à défendre » de Notre-Dame-des-Landes ou l'habiter comme politique
}

\author{
The "Area to Be Defended" at Notre-Dame-des-Landes, \\ or the Art of Inhabiting Places as Political Strategy
}

\author{
Frédéric BARBE
}

géographe, Centre de Recherche Nantais Architectures Urbanités, UMR Architectures Ambiances Urbanités 1563 , Ensa Nantes - 6 quai François-Mitterrand, BP16202, 44262 NANTES cedex 2. (frederic.barbe@crenau.archi.fr)

\begin{abstract}
Résumé : Le conflit socio-environnemental de Notre-Dame-des-Landes est actuellement l'un des plus importants conflits de ce type en Europe. Malgré sa longévité et sa complexité, malgré les coûts politiques et financiers qu'il engendre, malgré les formes d’innovation que l'on peut y trouver et les questions de gouvernance qu'il adresse à tous, ce haut lieu est peu enquêté par les sciences sociales. Dans la première partie, nous examinons le lieu du conflit et les conditions de la recherche dans ce lieu. Dans la deuxième partie, nous proposons d'élargir le périmètre habituellement considéré et d'offrir un récit réaliste de l'expérience de la "zone à défendre ». Ainsi, nous voulons montrer comment s'articulent la figure du haut lieu politique et des mises en réseau plurielles et à des échelles variées. Au travers de ces trois entrées, nous suggérons la réalité d’un « habiter en conscience d’habiter » comme ressource politique construite et partagée par des acteurs hétérogènes.
\end{abstract}

\begin{abstract}
The socio-environmental conflict at Notre-Dame-des-Landes is currently one of the most important eco-conflicts in Europe. Despite its unique characteristics - its long duration, its complexity, the high political and financial costs involved, the forms of political innovation generated, the questions of governance raised - the social sciences seem to have ignored this important place. First, we consider the place of conflict itself and the possibilities for honest social-science research that it offers. Second, we propose to go beyond the usual limits of the conflict area and describe how local arts of resistance combine different means of inhabiting the conflict area and generating counter-expertise. In this way, we wish to show how its status as a key political battleground and the associated multi-scale networks are interconnected. Through these three entry points, we hope to shed light on the day-to-day reality of "conscious inhabitation" of places as a political resource and strategy shared by heterogeneous actors.
\end{abstract}

Mots clés : habiter - contre-expertise - réseau - illégalisme - participation - ZAD

Keywords: sense of place - counter-expertise - network - illegalism-participation - ZAD (area to be defended)

Depuis l'automne 2012, la « zone d'aménagement différé » de Notre-Dame-des-Landes créée en 1974 est devenue la « zone à défendre », un territoire de 1650 hectares, singulier dans ses formes de résistance et de sécession, surmédiatisé et pourtant fai- blement étudié par les sciences sociales. Imaginé à la fin des années 1960 (Oream, 1971), le projet de construction d'un second aéroport international d'intérêt régional à proximité de Nantes est réactivé en 2000 sous le gouvernement Jospin et la déclaration 
d'utilité publique est actée en 2008. La construction de l'opposition à ce projet est inscrite dans cette longue durée et dans un espace multi-échelles. Trois générations d'habitants sont concernées. Ce que ses acteurs nomment "le mouvement » se réfère à de nombreux conflits proches ou lointains, actifs ou patrimonialisés : deux projets de centrales nucléaires en Basse-Loire, l'aménagement du bassin de la Loire, Donges-Est, Plogoff, le Larzac, et des conflits plus récents comme celui de Sivens, de Bure ou du Val di Susa, etc. Un premier livre de critique de l'aménagement et de la croissance, Dégage!... on aménage (Jean De Legge et Roger Leguen) est publié en 1976. Il cite Lacoste, édité la même année chez Maspéro, qui réinscrit la culture du conflit et du rapport de force dans la géographie. Le conflit d'aménagement de la décennie 1970 est devenu, quarante ans plus tard, un conflit socioenvironnemental complexe.

La réactivation du projet en 2000 s'accompagne d'une montée progressive des oppositions et de la formation d'un haut lieu politique déjà habité (ruraux et péri-urbains) et bientôt occupé (appel international à occupation par les premiers occupants, lors du Camp Climat de 2009). Depuis 2000 et surtout 2012 , les interventions variables de l'État et des grands exécutifs territoriaux comme la dilution du temps participatif ou judiciaire ont permis le développement d'une coalition marquée par la convergence de formes d'action diverses et la porosité entre groupes d'opposants très variés. Cette coalition hétérogène est large et efficace : à la date de finalisation de cet article, elle empêche toujours le démarrage des travaux et génère des alternatives pratiques et théoriques. Nous souhaitons ici contribuer à une connaissance objectivée de cette "zone à défendre ». Dans la première partie du texte, nous examinons les conditions de la recherche dans ce conflit. Dans la deuxième partie, nous montrons la nécessité d'élargir le périmètre d'étude habituellement considéré (celui de la ZAD) pour offrir un récit réaliste de l'expérience de la «zone à défendre ». Ainsi, nous montrons comment s'articulent un haut lieu politique et des mises en réseau plurielles et à des échelles variées. Dans ces trois propositions, nous proposons au lecteur de mobiliser le concept de «l'habiter » pour expliquer le comportement politique, relationnel et quotidien de très nombreux acteurs. Le mouvement contre ce projet d'aéroport est devenu un mouvement "contre l'aéroport et son monde ", mais il est aussi un conflit de lieu, inscrit dans la matérialité des relations à des territoires singuliers.

L'habiter est un concept qui apparaît régulièrement en philosophie et dans les sciences sociales, adaptatif et pluriel (Stock, 2004), très sensible aux modes intellectuelles et éditoriales. Il existe aussi sous d'autres appellations dans les mondes vernaculaires (Bonnemaison, 1986). Concept peu dense, mais très englobant, il est entendu dans cet article comme la relation sociale aux lieux. C'est un truisme de dire qu'il existe un très grand nombre de régimes d'habiter. Pour l'étude de ce conflit, nous proposons d'inventer et de mobiliser un « habiter en conscience d'habiter » comme un régime critique spécifique de l'habiter. Il engage la personne et le groupe dans le faire ensemble et le faire avec ce qu'on a et qui est là - ici et maintenant. En effet, « habiter » la ZAD et le «mouvement » pose des questions existentielles en permanence. On s'y déploie en tant que personne devant exercer sa souveraineté et membre de collectifs s'affirmant autonomes au milieu de tensions et de rapports de force variés et forts, dans une problématique géographique et environnementale quasi-permanente. Le danger (physique ou socioprofessionnel), la mise en danger de soi, le sentiment euphorique de l'inconnu, le plaisir parfois douloureux de la découverte de soi et de la libération de la puissance personnelle et collective par l'abandon des servitudes volontaires (La Boétie, 1547; Reich, 1933), la transgression individuelle et le multiplicateur transgressif de l'esprit de groupe excitent cette expérience de l'habiter. Nous proposons au lecteur de vérifier par lui-même cette hypothèse d'une contestation par "l'habiter en conscience d'habiter ». Ce régime critique de l'habiter serait une méta-ressource de nature politique, un habiter engagé, permettant de mobiliser l'ensemble des autres ressources des acteurs concernés. Loin de la croire réservée aux sociétés riches, je pense notamment la voir à l'œuvre chez les plus pauvres dans la théorie de la « démocratie profonde " (Appadurai, 2013), comme une forme politique élémentaire accessible à tous. 


\section{UNE PARTICIPATION OBSERVANTE ÉMANCIPÉE DANS UN CONFLIT SOCIO-ENVIRONNEMENTAL}

À partir de 2009 (premières résistances physiques aux forages menés par le concessionnaire), la « zone d'aménagement différé » est progressivement militarisée. La présence policière devient permanente en 2012-2013 pendant l'opération César et ses suites, paroxysme du conflit, début de destruction des lieux et tentative d'expulser les occupants par la force. La répression et la surveillance policières, mais aussi les pressions de toutes sortes touchent au même moment la métropole nantaise. Dans cette ambiance anxiogène, les conditions même de la recherche sont affectées par la tournure des événements.

\section{Historique d'une absence des sciences sociales}

Peu de travaux scientifiques ont été publiés sur ce conflit. Deux articles tardifs y sont consacrés (Renard et Rialland-Juin, février 2013; Pailloux, janvier 2015), quelques mémoires de master, des segments de thèse ou d'articles, notamment liés au bruit aéroportuaire, à la création de la Commission Nationale du Débat Public et aux chantiers participatifs. Un enseignant-chercheur nantais retraité nous signale en 2013 qu'un projet de Groupement d'Intérêt Public avait été évoqué dans les années 1980, puis abandonné en même temps que le projet. Je suis contacté par quelques doctorants ou collègues éloignés souhaitant venir sur la ZAD pour un terrain ou un atelier collectif. Cette atonie surprend, les seuls chercheurs rencontrés sur le terrain sont des étudiants de master, souvent géographiquement éloignés, engagés dans l'expérience même de la «zone à défendre », eux-mêmes en participation observante. Au regard de l'énorme production politique et contre-experte des différents acteurs de la coalition anti-aéroport, comme des massives « propagandes nécessaires »(De Legge, 2013) des grandes collectivités territoriales, les sciences sociales apparaissent invisibles. Même le grand colloque international de géographie rurale tenu à Nantes en 2014, intitulé « Les campagnes : espaces d'innovation dans un monde urbain » ne se déplace pas sur les lieux. Si nous voyons quelques collègues et aussi de nombreux étudiants nantais sur les lieux, ce ne sera jamais dans le cadre d'une recherche, mais dans celui d'un habiter des lieux.

Faute d'entretiens menés sur cette question, il est difficile d'expliquer cette absence autrement que par un ensemble d'hypothèses ainsi résumées : d'une part, l'idéologie de la compétition et de la métropolisation comme l'économie et la réorganisation de la recherche ne sont pas sans effet sur les objets que celle-ci se choisit; loin d'être neutre axiologiquement, le choix des sujets et des terrains de recherche est contrôlé par ses conditions politiques et financières. D'autre part, les modalités les plus courantes de la recherche, sans restitution intermédiaire, sans médiation, sans retour final aux acteurs et dont les produits ne sont pas librement accessibles, n'incitent pas à entrer dans des scènes conflictuelles revendiquant l'autonomie. En miroir de cette absence, de nombreux acteurs de la coalition anti-aéroport (et la zone à défendre ne diffère aucunement en cela de nombreux autres espaces sociaux) portent un regard premier distant, sinon hostile à l'enquête en sciences sociales. Dans ce conflit, en raison des tensions et de la surveillance policière, cette suspicion à l'encontre d'une recherche mal connue et jugée peut-être peu honnête, sinon complice d'une expertise aux ordres, nous a semblé particulièrement forte. Mais elle apparaît aussi paradoxale, puisque des chercheurs sont régulièrement invités dans les réunions publiques et les fêtes annuelles du mouvement. Le Collectif des naturalistes en lutte a pris cette question à bras-le-corps en expérimentant une recherche plus relationnelle. Selon nos observations, la participation observante que l'on pourrait décrire comme un régime d'habiter spécifique de la recherche, mais aussi la médiation du travail scientifique permettent de réduire la suspicion à son encontre.

\section{Une relation de recherche, la participation observante}

J'ai construit une situation de recherche, en partie rétrospective, depuis ce constat progressif de carence des sciences sociales et en me plaçant peu à peu dans une posture de « participation observante émancipée » (Soulé, 2007). Il s'agit d'abord pour moi de prendre en charge un travail que personne ne semble faire et cela se limite initialement au pro- 
jet de construire une archive de cette lutte avec laquelle j'interagis. S'il faut témoigner ici, et plus que dans une recherche classique, des conditions de la recherche, c'est aussi parce que la participation observante reste encore aujourd'hui suspecte. Dans mon cas, ce mode de recherche exigeant combine des temps d'action et de distanciation, au sein de la coalition des opposants. Son biais principal semble être ici l'absence d'enquête régulière auprès des porteurs du projet. Ce travail suppose à un certain moment d'afficher clairement auprès de certains interlocuteurs le projet d'écrire sur la ZAD sur un registre académique, notamment en vue des entretiens et des relectures de versions successives de cet article par des acteurs de la coalition. Mon entrée dans le conflit date de 2009, lors de la préparation du Camp Climat (premières réunions à la Vache Rit, le hangar agricole lieu de réunion historique du mouvement) et du Camp Climat lui-même, où je suis présent avec une association nantaise, des livres, une tente, des ami-e-s. Habitant Rezé, une des trois communes du Sud-Loire riveraines de l'aéroport international Nantes-Atlantique, j'ai longtemps travaillé dans le collège de Bouguenais, dernier bâtiment de hauteur dans le couloir d'atterrissage de l'aéroport. Je connais et utilise avec mes élèves d'alors le premier document de contre-expertise contre le transfert, édité et diffusé par la mairie de Bouguenais aux habitants de la commune en 1997, De Bouguenais à Notre-Dame-des-Landes, un transfert à haut risque. En 2000, nous manifestons, chantons et tapons même sur des casseroles en compagnie d'élèves et de parents d'élèves dans le hall de Nantes-Atlantique, mais c'est contre Claude Allègre, alors ministre de l'Éducation nationale.

D'emblée, et je montrerai plus loin la nécessité de cet élargissement du périmètre d'intérêt, mon « habiter» du conflit comme géographe et habitant se déploie tout autant au Sud-Loire qu'à la ZAD. Les actions-observations se déroulent sur les deux terrains : libres divagations, réunions du mouvement, inter-collectifs régionaux ou nationaux, ateliers, fêtes et manifestations sur la ZAD, mais aussi à Nantes, dans les trois communes riveraines de l'aéroport et dans le pays de Retz, en général plus près de la dynamique organisatrice. En 2011, je participe à l'écriture et l'édition du second livre sur le conflit, C'est quoi c'tarmac, profits, mensonges et résistances (Collectif Sudav, 2011) écrit depuis les deux territoires, ZAD et Sud-Loire, puis en 2014-2015 à l'émergence du groupe CartoZ. L'engagement dans une telle démarche scientifique vient d'abord d'un attrait certain pour « l'empirisme radical » porté notamment par Becker ou Peneff, son passeur français. La lecture de L'expérience de terrain (Mohia, 2008) ou d'Arjun Appadurai (2013), de sa «démocratie profonde » et de son «droit à la recherche » m’ont aidé à penser une recherche relationnelle, éloignée de toute colonialité. Biau et al. (2013) ont montré a contrario combien la plupart des formations disciplinaires présupposent, enseignent et s'autojustifient par l'incompétence présumée des personnes ordinaires. Le travail de recherche et d'édition du Centre d'Histoire du Travail de Nantes sur l'histoire sociale du département m'a également nourri, sur le fond, mais aussi sur la manière habile et souvent touchante dont de nombreux acteurs engagés géraient leurs archives et passaient à la recherche et à l'écriture. La participation observante est ce qui me paraît nommer le mode de recherche utilisé ici. En la qualifiant d'émancipée (Soulé, 2007 ; Rancière, 1987 et 2008), je dis qu'elle s'est constamment accompagnée d'un processus d'objectivation des situations, mais aussi d'une très grande tolérance personnelle à la diversité des acteurs et des expériences, qu'elle est une forme maîtrisée de " passion cognitive » (Roux et al., 2009). Elle conjugue ainsi « la pensée par cas » (Passeron et Revel, 2005) et « la reprise de l'affectivité ", du contradictoire et du sensible dans la recherche (Desprest, 2009).

\section{L'habiter d'une lutte}

En important les concepts de topos et de chôra (Berque, 2000) dans le contexte d'un conflit d'aménagement ayant changé de nature, nous souhaitons rappeler que la relation au lieu (la relation d'un groupe dans et à une portion d'étendue terrestre) est une composante difficilement contestable de ce conflit. Au lieu mathématisé de l'aménageur (topos) et de l'élu visionnaire, est substitué, articulé, arrangé, opposé par une grande partie du mouvement, le lieu existentiel et relationnel (chôra), souvent figuré dans la littérature des occupants comme «zone d'autonomie temporaire » (Bey, 1997) et de plus en plus «Commune ». L'habiter ainsi tourné est l'art d'être habitant d'un lieu, indigène. Mais cet habiter du 
mouvement décrit aussi la manière de circuler et de s'approprier de nouveaux lieux, inconnus, et la sensibilité de «la $\mathrm{ZAD}^{1}{ }^{1}$ » au fait urbain et au fait migratoire, à l'internationalisme, en témoigne : « la ZAD » fournit régulièrement de la nourriture aux deux squats de sans-papiers de Nantes et à des habitants d'origine roumaine des bidonvilles de l'agglomération; en mars 2016, « des zadistes » sont présents lors de l'expulsion d'un grand bidonville et participent au déplacement des caravanes délabrées des expulsés. L'habiter du mouvement contre l'aéroport et son monde semble alors relever d'une synthèse de figures souvent opposées, celles de l'indigène et du migrant, du rural et de l'urbain : une combinatoire psycho-géographique (Moles, 1992) datée et située, glocalisée. En effet, cet habiter s'inscrit aussi dans l'histoire sociale paysanne et ouvrière (et celle de l'économie sociale) de la Basse-Loire, vécue directement par de nombreux acteurs âgés et retraités qui constituent une part active du mouvement. Transmise aux plus jeunes par des récits d'imprégnation lors des moments collectifs, elle est explorée et mise en scène par certains groupes d'occupants (comme le Collectif Mauvaise troupe). Cette intégration de l'expérience de la zone à défendre dans l'histoire socio-spatiale régionale (Lambert, 1970; Le Madec, 1988; Bourrigaud, 1994; Barbe, 2008) explique aussi la prise de position de la CGT (CGT, 2015) contre le projet d'aéroport, qui était loin d'être acquise en raison de sa tradition productiviste. Enfin, les liens noués avec d'autres luttes ici et ailleurs (THT, Sivens, Bure, Val di Susa, Rosia Montana, différents États du Mexique, Ferme des milles vaches, etc.) reprennent un répertoire de solidarité et de formation mutuelle des années 1970 et 1980 (Plogoff, le Larzac, le Nicaragua sont alors reliés à la Loire-Atlantique) et mobilisent ensemble différentes catégories d'opposants.

Cet habiter de résistance collective nourrit et se nourrit d'une critique de la modernité occidentale et des modes d'aménager issus notamment de la modernité stato-nationale et coloniale, puis de la mondialisation néo-libérale structurant une nouvelle compétition territoriale. Il nous semble

1. L'emploi de ce géographisme ou sociologisme (selon les cas) est signalé ici volontairement pour rappeler au lecteur un usage courant mais contextuel du terme : « on va à la ZAD », « la ZAD peut amener des légumes et des plants à repiquer ». On comprendra qu'il s'agit parfois du lieu, d'autres fois des occupants dans le lieu ou ailleurs, le plus souvent d'un mot à large spectre, que seul le contexte d'énonciation précise. constituer la ressource principale et bon marché des habitants et occupants. Habiter en conscience d'habiter, c'est faire de la politique avec son corps, avec sa vie. C'est être un, relié aux autres et aux lieux. En tant que ressource relationnelle, l'habiter en conscience d'habiter (qui nous semble une forme de conscience politique particulière investissant la relation aux lieux) permet de mobiliser de nombreuses autres ressources de toutes natures. Dans certaines sensibilités habitantes, nous voyons des formes discrètes d'autochtonie associées dans la lutte à un grand internationalisme : des paysans nés sur place refusent le concept étroit de propriété et se disent " passeurs de terre » de génération en génération, engagés dans des luttes sociales depuis les années 1970 et choqués par la catégorisation de «mauvaises terres » véhiculée par les porteurs du projet. Des occupants très jeunes, venant parfois de pays étrangers, très mobiles, fonctionnant en réseau étendu, disent leur attachement au nouveau lieu. La mobilité paysanne est également forte, chaque manifestation en rend compte. Cet habiter spécifique offre des ressources pour échapper au syndrome et au stigmate "Nimby » décrivant l'enfermement égoïste des riverains s'opposant à l'intérêt général. Habiter politique usant de références très hétérogènes, croisant et expérimentant des formes d'organisation diverses, il échappe également au risque populiste d'une souveraineté critique réduite à l'empêchement (Rosanvallon, 2006). Démentant le mythe du citoyen passif, ce mouvement « contre l'aéroport et son monde » porte aussi des propositions et des alternatives. L'habitant n'est manifestement pas le riverain, terme de plus en plus accaparé par la pensée de l'exclusion (Fassin et al., 2014). Localement, la catégorie "riverains » (les habitants des communes impactées par le projet ou les automobilistes les traversant régulièrement) est mobilisée par les porteurs du projet pour les décrire comme victimes quotidiennes des occupants dits radicaux de la ZAD et appeler à une action répressive, elle-même annoncée explicitement radicale.

L'intensité et la singularité de ce conflit faiblement étudié mettent en lumière les relations complexes entre la recherche et l'habiter des terrains de la recherche. Nous voyons aussi dans cet évitement académique la trace d'un nœud épistémologique et éthique au sein même du tournant participatif initié par l'État et les collectivités (Mohia, 2007 ; Peneff, 
2009 ; Collignon, 2010; Volvey et al., 2012; Biau et al., 2013). L'habiter en conscience d'habiter porte une dynamique de recherche, un véritable « droit à la recherche » des habitants (Appadurai, 2013).

\section{L'HABITER ET LA CONTRE-EXPERTISE}

La perception commune des échelles du conflit a fortement évolué au cours du temps. Plutôt enfermé entre 2000 et 2012 dans l'étroit périmètre administratif de la ZAD, le conflit a connu une série d'extensions : à la métropole nantaise, à l'Europe, puis à la France entière lors de la phase «ZAD PARTOUT », puis plus spécifiquement à la partie de l'agglomération riveraine de l'aéroport international NantesAtlantique et, à nouveau, à l'international, etc. L'existence préalable d'un aéroport international reconnu par le monde professionnel, sûr, profitable (aujourd'hui privatisé et géré par le concessionnaire du projet de nouvel aéroport) et modulable, ne peut être ignoré dans l'analyse de ce conflit. Il s'agit donc d'abord de relier ces deux territoires par l'enquête.

\section{L'endroit et l'envers d'un conflit : relier deux « habiter»}

L'endroit, c'est la zone à défendre. Aller sur la ZAD, à pied, à vélo, en stop, en voiture. Y dormir parfois, dans une tente en plein été, dans une chambre de ferme surpeuplée pendant la garde hivernale d'un lieu menacé de destruction. Divaguer encore, parcourir les chemins, éviter les checkpoints de la gendarmerie mobile en traversant des prairies et des champs détrempés, être accueilli dans un lieu, puis un autre, travailler, lever les suspicions, rencontrer des gens et des métiers que l'on ne rencontrerait pas, amener des livres, de l'argent, de la nourriture, en ramener de la ZAD à d'autres moments vers la ville. Lire. Y lire Erri de Luca sous un chapiteau. Reconnaître des acteurs peu à peu médiatisés et pourtant discrets, voire anonymés pendant la phase des «Camille». Écouter. Prendre l'apéro. Faire des inventaires naturalistes en bande organisée, accoucher d'un débat sur l'avenir de la ZAD après l'abandon du projet malgré les craintes de certains acteurs que cela démobilise, faire des cartes, les éditer, les vendre par milliers en un seul jour sur une quatre-voies piétonnisée par force, faire une conférence au Festival International de
Géographie de Saint-Dié-des-Vosges, participer aux ateliers d'écriture du Journal Intime Collectif. Être contrôlé par la gendarmerie, à pied, en vélo, en voiture, en stop. Manifester. Être photographié depuis le sol ou depuis un hélicoptère, en train de manifester, de se réunir, de ramasser des pommes de terre ou de semer du sarrasin. Rencontrer des autochtones et leurs petites-grandes histoires, faire «visiter» la ZAD à des Maliens, des Lyonnais, des timides, des inquiets ( «la ZAD» est fréquemment décrite par les pro-aéroports et les médias comme une zone de non-droit, une organisation terroriste, un mouvement armé avec sa vitrine légale), à ceux qui finalement demandent à voir. Être un indigène au pluriel. Croiser des journalistes dissidents, des étudiants qui nous demandent pourquoi on n'en parle pas à la fac, des sans-papiers africains réfugiés sur la zone et qui colorent d'un coup cet univers très blanc de peau.

Cet endroit singulier, ce "Las Vegas » paysan, squatter, péri-urbain, communaliste et indigéniste, a un envers, c'est le Sud-Loire, son aéroport international, ses nuisances sonores, ses zones d'activités et ses emplois liés, ses habitants qui font moins parler d'eux. À Bouguenais et Rezé, j’observe également la différence entre la catégorie « habitant » et la catégorie « riverain ». L'absence d'associations consistantes d'habitants luttant contre le bruit aéroportuaire et l'activité des plus réduites ${ }^{2}$ de la Commission consultative d'environnement de Nantes-Atlantique confirme "l'influence du sentiment d'appartenance territoriale dans la délimitation du périmètre géographique de gêne sonore » en montrant également comment «le vécu du bruit des avions [peut], en forgeant des pratiques et représentations spatialisées, alimenter l'identité locale dès lors en tension avec la symbolique d'altérité spatiale, sociale et politique que l'objet « bruit des avions » véhicule» (Faburel, 2003a et b). Si les habitants du Sud-Loire avaient réclamé activement la fermeture de l'actuel aéroport, le nouveau serait certainement déjà construit. La figure de l'habitant plutôt populaire et pragmatique, conscient des externalités positives de la zone aéroportuaire actuelle et des risques que le transfert fait courir aux stratégies familiales (perte d'emplois, majoration des temps et des coûts de transport, déstructuration et gentrifi-

2. [http://www.acnusa.fr/fr/les-aeroports/aeroports-acnusa/nantes-atlantique/31]. 
cation des espaces de vie) semble alors très éloignée de celle, étroite, du riverain réduit à une seule donnée mesurable (la distance ou le bruit « objectif »). L'actuelle zone aéroportuaire est aussi un bassin de vie et d'emploi. L'encadré ci-dessous rend compte de l'activité du Collectif de Rezé contre le projet d'aéroport à Notre-Dame-des-Landes, un collectif d'habitants créé fin 2012.

Cet inventaire dit mal le fait que l'action du collectif rezéen s'est toujours liée dans l'action à la dynamique de la ZAD et que ses membres ont toujours défendu « l'expérience de la ZAD » lors des rencontres avec les habitants, les élus, les journalistes, y compris dans les moments de tension et de pression politique extrêmes, et notamment autour de la grande manifestation du 22 février 2014, lorsque les élus porteurs du projet et le préfet recourent de manière récurrente et sans nuances aux catégories du terrorisme pour intimider, trier et diviser les opposants au projet. C'est ce lien entre un endroit, la ZAD «extra-ordinaire » et un envers, le Sud-Loire " ordinaire », qui relocalise à mon sens le conflit. Ce lien n'a été reconnu que progressivement au sein du mouvement et c'est la pluralité de celui-ci qui a permis notamment que se réalise en 2014-2015 le travail de l'Atelier citoyen, un «bureau d'études » éphémère et collaboratif pour l'optimisation et la bonne gestion de l'actuel aéroport, aujourd'hui concédé au groupe Vinci. Incarnant les liens humains, politiques et quotidiens entre les deux territoires, l'ancienne maire et conseillère générale socialiste de Bouguenais est devenue l'une des figures publiques du mouvement contre l'aéroport et son monde (Verchère, 2016). Les nouvelles oppositions municipales de gauche à Rezé et Bouguenais comme les prises de position de la CGT locale, départementale, régionale, enfin nationale et du groupe Vinci lui-même (au terme d'un long processus 2011-2015) sont également fondées sur ce lien entre les deux territoires - des territoires « circulants ».

\section{L'expérience de la zone à défendre de Notre-Dame-des-Landes}

La zone était habitée avant 2009. L'opposition au projet datant du début du projet (1972-1974), ce sont ces habitants à la fois ruraux et péri-urbains qui détiennent l'historicité et la légitimité de la lutte. Mais c'est aussi grâce à l'occupation et aux occu-

- Réunions hebdomadaires 2012-2015, puis mensuelles, dans un café.

- Discussions, auto-formation, délibérations.

- Participations à de nombreuses actions de soutien, manifestations, inauguration de gare, hommages.

- Accueil et hébergement de la marche de Nice en 2013 et autres événements de même type.

- Tractage et collage sur Rezé et Nantes (à pied, en voiture et à vélo).

- Interpellations des élus (candidats aux municipales, député Raimbourg) (2013-2014).

- Communiqués de presse (une dizaine) rarement diffusés.

- Participation aux réunions inter-collectifs nationale et Sud-Loire, aux réunions de préparation de la manifestation du 22 février à NDDL et à Nantes (2014).

- Organisation d'une réunion publique sur les grands projets dans l'agglo nantaise (2014).

- Organisation d'un forum «Quel devenir pour la ZAD en cas d'abandon du projet ? » lors du rassemblement antiaéroport de juillet 2014.

- Organisation de «l'Assemblée de Rezé » le 20 septembre 2014 autour de l'appel pour les libertés publiques lancé à l'initiative du Collectif, participation à ses suites nombreuses pour la convergence et la pluralité des luttes, contre la répression.

- Participation de plusieurs personnes du Collectif à l'Atelier citoyen Nantes-Atlantique.

- Organisation d'une grande réunion publique (400 participants, novembre 2015) sur les propositions de l'Atelier citoyen à Saint-Aignan-de-Grandlieu.

Inventaire des principales actions du Collectif de Rezé contre le projet d'aéroport à Notre-Dame-des-Landes (une association de fait, sans bureau, ni président, ni compte bancaire), extrait de la mailing-list du collectif - printemps 2015, complété printemps 2016 (deux dernières lignes)

Llist of the main actions undertaken by the Collectif de Rezé Contre le Projet d'Aéroport à Notre-Dame-des-Landes ("Rezé Collective Against the Notre-Dame-des-Landes Airport Project", an informal association with no office, board, chairperson or bank account), taken from the Collective's mailing list - spring 2015 (spring 2016 for the last two lines) 
pants, à leur interposition physique face à la police, que le projet est arrêté et la zone toujours habitée : ils ont aussi acquis leur propre légitimité. Cette expérience ne saurait donc être réduite à la seule présence des occupants, mais bien à une interaction entre des habitants dits « historiques » et des occupants néoruraux. Ceux-ci sont souvent nommés «zadistes » à partir de 2012, une appellation commode, mais jugée souvent réductrice et inappropriée, voire malveillante par ceux et celles qu'elle désigne. L'usage de ce terme semble de fait avoir décliné dans le mouvement au profit des appellations « occupants », « jeunes » ou encore « gens de la ZAD ». Au-delà de certaines tendances (jeunesse, sur-masculinité, peu de personnes issues de la diversité, des histoires familiales souvent engagées), je rapporte un sentiment d'hétérogénéité. La diversité des occupants est pourtant quelquefois réduite au sein même de la ZAD à quelques figures d'opposition non superposables : « historiques » et « arrivants », «politiques » et « arrachés » (au sens de punks, routards et autres dénominations associées), ceux qui ont des projets agricoles et les autres. Pour un visiteur, il me semble que c'est la jeunesse et le sentiment de rupture volontaire avec l'ordre du travail capitaliste et bureaucratique (relation au temps, au projet, à l'ambition) qui est le plus frappant. Des occupants parmi les plus anciens (certains ont déjà quitté la zone pour des raisons de fatigue ou de menaces judiciaires) présentent la ZAD comme un lieu de vie et d'auto-formation par le collectif, sans aucun romantisme et quelquefois en se moquant sévèrement des projections fantasmatiques des personnes ne vivant pas sur la ZAD. De fait, c'est un lieu circulant, poreux. La ZAD vit avec et de l'extérieur, cet extérieur qui lui assure les conditions de sa survie (circulation des occupants, afflux de militants témoins ou actifs à chaque intervention policière, nombreux coups de main et dons de toutes sortes) et quil nourrit en retour de quelque chose. En cela, la ZAD ne diffère guère d'autres expériences alternatives telles les territoires zapatistes (Marie, 2011) soutenues de l'extérieur et procurant à ceux de l'extérieur une référence politique et géographique. La lutte a généré un paysage et un style relationnel. La D281, une départementale fermée et sortie du réseau routier par le Conseil départemental, rouverte par les opposants et encombrée de chicanes, constructions et traces de lutte (épaves, feux, fossés, graffitis) est à la fois un paysage post-apocalyptique et la piétonisation informelle d'une partie de la ZAD, aujourd'hui très habitée. Les réunions mensuelles ou hebdomadaires du mouvement à la Vache Rit, le long de l'autre départementale, permettent de mieux comprendre comment les acteurs arrivent à coopérer dans la lutte. Malgré leur longueur, leur lenteur, leurs imbroglios parfois, ces réunions bien régulées ont «marché » contre toute attente, produisant de la décision en contexte de crise et, pourrait-on dire, en contexte interculturel, tant les acteurs semblent parfois éloignés les uns des autres. Cette culture de la délibération, lente, chaotique, non sans coups de colère et manœuvres, est très éloignée de la culture faiblement délibérative inculquée par l'École, l'État et les institutions. Ces méthodes alternatives, en partie transmises sur la $\mathrm{ZAD}^{3}$ semblent donc porteuses d'une certaine efficacité. La présence régulière de «petits élus » (eux-mêmes regroupés dans une association spécifique d'un millier d'élus, le CéDpa ${ }^{4}$, créé en 2009) a également permis que la représentation politique y reste constamment présente, sans toutefois réussir une médiation politique du type de celle de Toulouse ${ }^{5}$. La dynamique croisée de résistance et de sécession produit alors une culture de la contre-expertise, de l'action directe et de l'illégalisme, culture négociée, fragile, mais performative.

\section{Deux « habiter » paradoxaux : celui de l'ingénieur, celui du naturaliste}

Mais l'expérience de la ZAD, c'est aussi des approches plus inattendues, loin du cliché zadiste ou paysan, comme celle de l'association Nexus ou des Naturalistes en lutte. Nexus est une association créée en 2010 pour « informer les citoyens et les acteurs économiques de l'Ouest Atlantique des enjeux du transport régional à moyen et long terme ». Politiquement, elle est proche du Modem ${ }^{6}$ (parti

3. Voir, par exemple, le texte «Débat sur les débats » (anonyme) [https:// infokiosques.net/spip.php?article87].

4. [http://aeroportnddl.fr/].

5. Le projet de transfert de l'aéroport de Toulouse-Blagnac, lancé en 2001 après la catastrophe d'AZF, est officiellement abandonné par l'État en mars 2013. Si le préfet de région utilise les mêmes types d'arguments et d'alternatives que les opposants à NDDL pour justifier l'abandon du projet, c'est d'abord l'existence d'une forte opposition politique pendant le temps des études (2001-2006), entre la métropole toulousaine porteuse du projet, alors à droite (jusqu'en 2008) et les autres exécutifs à gauche, qui empêche la formation d'un consensus des exécutifs locaux.

6. Leur leader national, venu sur la ZAD lors de la campagne des régionales de 2010, rencontre les élus opposés au projet, prend le café chez un exploitant historique en lutte et s'exprime contre les excès de la métropolisation en demandant que tout le processus soit repris. 
politique membre de la Coordination anti-aéroport) dont des militants et des élus constituent le conseil d'administration, mais elle est aussi appuyée sur une compétence transport singulière, celle d'un ingénieur transport à la retraite ${ }^{7}$, habitant à proximité de la ZAD. Nous voudrions montrer comment cette double affiliation au centrisme le plus légaliste et au monde productiviste de l'ingénieur n'empêche pas Nexus d'être également proche du régime d'habiter de la ZAD. Sa tentative indépendante de reformuler l'intérêt général semble esquisser une forme de « décence ordinaire » (Michéa, 2011) appliquée aux politiques publiques. Ici, l'habiter en conscience d'habiter nous apparaît comme une transversalité susceptible d'être activée partout, même au sein de la rationalité mathématisée de l'ingénieur et au-delà des contradictions propres à chaque posture. Nexus « habite » bien le conflit, au sens où celui-ci est effectivement un ensemble de lieux et de relations (visites, réunions et démonstrations). En y mobilisant une parole contre-experte appuyée sur une carrière d'expert (entretien, 2012), Nexus est un contradicteur important et théâtral qui a ridiculisé à plusieurs reprises l'action publique en matière de transports collectifs ${ }^{8}$ et montré une grande inventivité. Le conflit de Notre-Dame-des-Landes prend souvent la forme d'une scène ou d'un ensemble de scènes reliées, dans lesquelles la confrontation des modes d'expression met les questions de créativité et d'art au centre - où les dimensions subjectives du vécu dépassent le plus souvent les données dites objectives. Certains occupants en font profession de foi (Collectif ZAD Partout, 2013). À sa manière conforme et légaliste, l'association Nexus participe à cette scène et retourne, à travers un effet miroir, une vision critique et politique de l'aménagement du territoire. Elle ouvre également un espace de création, celui de l'ingénieur libéré du commanditaire, redevable du seul intérêt général et de sa «folie » créatrice d'habitant souverain et outillé.

Le Collectif des naturalistes en lutte ${ }^{9}$ est composé d'universitaires, de professionnels et d'associatifs

\footnotetext{
7. Il tient un site à partir de 2008 « ESG INFRA, consultant énergies renouvelables transport de masse », qui préfigure l'action de l'association Nexus [http://www.esginfra.fr].

8. Par exemple, en attaquant au tribunal administratif la commande par la Région Pays de la Loire des rames de tram-train pour la réouverture de la ligne Nantes-Chateaubriand dix-huit mois avant le début de l'enquête publique, rendant caduque le nécessaire débat public sur la préférence accordée au tram-train aux dépens du Ter.

9. [https://naturalistesenlutte.wordpress.com].
}

du Grand Ouest. Même si certaines figures sont connues, comme l'ancien directeur de la réserve naturelle de Grandlieu, l'expression collective est la règle. La question de la nature évolue et les Naturalistes en lutte tentent d'adapter leurs pratiques et leurs représentations (entretien, 2014). Il s'agit d'étendre une méthodologie testée dès 2009 par l'association Bretagne Vivante lors de « défis pour la biodiversité » et documentée ailleurs (Manceron, 2015). Ces opérations permettent de réaliser l'inventaire de la biodiversité d'un territoire par un maximum de spécialistes, professionnels ou non, en dehors du temps de travail et dans une ambiance de démonstration plutôt conviviale. À Notre-Damedes-Landes, l'idée d'approfondir cette méthodologie se cristallise fin 2012 après l'opération César. La première sortie collective a lieu en janvier 2013, la participation est totalement inespérée et demeure forte tout au long du processus. C'est le premier usage d'une telle méthodologie en contre-expertise en vue d'une étude complète (espèces, habitats naturels, sols et sous-sols, classement des talus et hydrologie) dans un paysage non métropolitain peu transformé depuis les années 1970. Les objectifs poursuivis sont nombreux : refaire l'inventaire réalisé par le bureau d'études mandaté; faire évoluer les connaissances sur un type de paysage rural autrefois ordinaire et devenu exceptionnel; nourrir de possibles actions de recours juridique; alimenter le débat interne de la profession sur la compensation, nouveau levier de l'aménagement et dont le projet de nouvel aéroport constitue un laboratoire; enfin, mettre en valeur la biodiversité dans la société, dans une nouvelle forme d'éducation populaire au sein même d'un conflit environnemental. « Il y a eu ce désir de passionner les gens, même si c'est plus long avec les gens ", le Collectif " a fait de la politique sans le savoir » (entretien, 2014). Le bilan tiré est « une heureuse surprise ». Le territoire du projet apparaît d'une richesse exceptionnelle à l'échelle du département. Il constitue un carrefour biologique à la croisée sommitale de trois bassins-versants, non compensable car assurant des fonctions de connectivité contre les dynamiques d'extinction. Dans ce désir de populariser la nature, l'interaction en réunion a été parfois rugueuse avec d'autres acteurs, mais a favorisé l'acceptation partagée d'une "nature » secondarisée (ce paysage rural date de la révolution agricole du XIX ${ }^{e}$ siècle) digne d'intérêt (Godet, 2012). L'habiter 
est ainsi étendu à une critique et à une ré-invention de la méthodologie environnementaliste, davantage relationnelle et reliée aux enjeux politiques. Il vient également questionner la frontière entre humains et non-humains dans une nouvelle perception de la naturalité des paysages fortement anthropisés. « La sensibilité écologique » ou "sensibilité aux nonhumains » ici détachée de la sphère électorale (les groupes politiques écologistes sont une composante parmi d'autres du mouvement) nous semble évoluer vers une perspective de bien commun à construire et négocier.

En reliant la ZAD et la zone de l'aéroport NantesAtlantique, nous rappelons naïvement au lecteur qu'une situation ne s'explique pas uniquement dans le lieu même où elle se produit. Différents lieux et habiters sont concernés, les formes de lutte sont proliférantes, les circulations importantes, les excroissances inédites. Cette dynamique véritablement darwinienne (Moretti, 2008) traduit le très large spectre de la coalition. Il y a de la place pour tout le monde. Il n'y pas de chef et la contradiction est intégrée. Les nombreuses productions artistiques, politiques et contre-expertes du mouvement (Kempf, 2014) occupent l'espace vide des sciences sociales. Le régime d'habiter en conscience d'habiter réunit les acteurs du mouvement dans l'expérience commune des rapports de force construits ensemble contre les autorités.

\section{LE HAUT LIEU ET LE RÉSEAU}

Nous espérons amener le lecteur à appréhender l'habiter de la ZAD comme un faire ensemble dans les lieux, mais aussi un faire avec (ce qu'on a, qui est là) dans un lieu devenu extra-ordinaire, un haut lieu relié et circulant. Nous y observons notamment la figure du chantier collectif, geste de l'habiter ouvert aux coups de mains extérieurs, de «l'appel d'offres » de la ZAD (Pruvost, 2015). Nous observons la création de Sème ta ZAD, collectif d'exploitants et d'occupants désireux de mettre en pratique une agriculture humaniste et l'autonomie alimentaire de la lutte. Des terres expropriées d'exploitants ayant accepté l'indemnisation sont remises en culture dans une pratique collective de mobilisation, d'apprentissage et de production bien cartographiée. Sème ta ZAD naît aussi des occupations et défenses communes de certains lieux emblématiques sous menace policière et donc dans un habiter qui est celui du faire ensemble en urgence, expériences à très forte empreinte relationnelle (nuits de veille partagées, menaces physiques). L'habiter en conscience d'habiter est un habiter de l'interconnaissance active et de l'action directe que le retrait de l'État en 2013 a laissé prospérer : il faut gérer ensemble l'état des routes, des fossés, les déchets, reconnecter la ZAD fragmentée pendant l'occupation policière, (piétonisation, (re)construction, réparation de vélos, radio, journal interne, boulangerie, maraîchage, "non-marché », épicerie, etc.). Mais la ZAD, c'est aussi la grande association de la lutte, l'Acipa, créée en 2000, avec ses réseaux militants bien organisés, sa "force de frappe », ses figures expérimentées et son formalisme loi 1901. C'est aussi l'association historique des exploitants impactés, l'Adeca, beaucoup plus ancienne ${ }^{10}$, dans laquelle l'agriculture paysanne est incarnée par des hommes et des femmes nés ou installés adultes sur la zone. Ces deux associations ont accepté la venue des occupants et les ont soutenus. L'observation directe montre que des liens interpersonnels se sont noués au fil du temps et que les acteurs se sont apprivoisés mutuellement, au-delà de leurs contradictions et des déceptions croisées qui persistent. Ces processus ont transformé la zone dans un habiter fortement et obligatoirement partagé au regard des conditions de la lutte. Devenue un haut lieu, la zone à défendre voit sa géographie évoluer.

\section{Le haut lieu, une figure géographique}

Depuis les premiers jours de l'occupation de la ZAD, des cartes de toutes sortes ont été produites ${ }^{11}$, tantôt manuscrites, tantôt sous forme d'extraits annotés de cartes Ign. Une carte est utile pour circuler et s'approprier rapidement le territoire. Quelquefois aussi pour se perdre. Depuis deux ans, un groupe informel réunissant des occupants et des habitants de la métropole nantaise développe un système d'information géographique portant notamment sur la question foncière. Cette approche par la carte pose concrètement la question du devenir de la ZAD après l'annulation éventuelle du projet : devenir des terres expropriées, devenir des occu-

\footnotetext{
10. Certains de ces exploitants ont toujours connu le projet, lancé lorsque leurs pères étaient exploitants des mêmes terres.

11. Un certain nombre sont disponibles sur le site internet des occupants.
} 
pants, devenir de l'habiter en conscience d'habiter, devenir de l'expérience. La cartographie outille ainsi l'engagement en six points ${ }^{12}$ pris en novembre 2015 par le mouvement quant au devenir de la ZAD en cas d'abandon du projet (Collectif Mauvaise Troupe, 2016). Le groupe CartoZ montre les limites de la technophobie des occupants : un Sig est utile au projet de «communs » qui pourraient naître de l'abandon du projet, il est mis en œuvre. Nous proposons au lecteur de parcourir la ZAD en cinq cartes qui éclairent la complexité du territoire et du conflit.

Nous observons d'abord (figure 1) que la ZAD est située hors de la métropole, à une trentaine de kilomètres de la ville, bien que le projet soit principalement soutenu par l'exécutif métropolitain. Les travaux projetés (figure 2 - planche IV) mettent en évidence un dimensionnement de l'équipement à deux pistes, une zone économique à l'ouest, une zone de compensation à l'est et des infrastructures d'accès. La carte des lieux de vie des occupants (figure 3 - planche V) évoque une ZAD de l'Ouest et une ZAD de l'Est, longtemps dissociées par l'occupation policière. La carte agricole (figure 4 planche VI) montre qu'une majorité des terres agricoles sont aujourd'hui exploitées soit par les paysans en lutte, soit par les occupants, soit par le collectif paysans/occupants Sème ta ZAD. Enfin, la carte sensible de la ZAD (figure 5 - planche VII), éditée en février 2016 à 5000 exemplaires, vendue en quelques semaines et rapidement rééditée, se range dans les cartes subjectives (Barbe, 2015). Faite pour se perdre dans la ZAD, elle est aussi un récit cartographique. L'attachement mêle ici poésie, beauté et expérience intime des lieux.

J'ai travaillé précédemment (Barbe, 2014) sur les figures multiples du haut lieu apparu contre toute attente, c'est-à-dire du haut lieu statistiquement dérisoire et périphérique. Nous avons notamment comparé les îles Aran avec le pays dogon et le Chiapas. Nous retrouvons dans la ZAD les grands principes structurant le haut lieu périphérique : une alliance variable entre indigènes et visiteurs, une série de points d'intérêts politiques (incluant parfois la conflictualité), une série de styles et de médias possibles et accessibles, une dimension multisca-

12. Droit à rester sur place, continuité des exploitations agricoles, maintien des expérimentations des occupants, création d'un commun des terres expropriées, résolution collective des conflits nés de la mise en œuvre des points précédents. laire, une spirale de notoriété et d'attractivité grandissantes. Le Chiapas est l'exemple qui nous semble le plus proche de la ZAD : une alliance entre des groupes indigènes et des néo-ruraux très politisés, une politique de développement néo-capitaliste en contexte autoritaire et mafieux, l'anonymat, un accès aux nouvelles technologies et à des formes d'écritures mondialisées, des revendications locales et internationales à l'intérieur du cadre national mexicain, des circulations et un «tourisme " militant protecteurs et profitables. Toutes choses égales par ailleurs (et notamment la taille, l'historicité, l'interculturalité et les degrés très différents de violence de l'environnement), le processus semble identique sur la ZAD de Notre-Dame-des-Landes, y compris dans les effets d'échelles, de réseau et de diffusion.

\section{Comment le haut lieu résonne dans les territoires proches}

Nous observons deux formes de mise en réseau, celle d'agriculteurs habitant dans un vaste périmètre autour de la ZAD d'abord, celle de listes citoyennes dans la métropole nantaise ensuite. Précédé d'Assemblées paysannes, le Collectif des Organisations Professionnelles Agricoles Indignées par le projet d'aéroport (Copain) est véritablement créé en 2011. Décliné par département, il est proche de la Confédération paysanne sans être soumis à la logique électorale du syndicat paysan, né pour partie en Loire-Atlantique et qui en a administré la Chambre d'Agriculture jusqu'en 2007. L'objectif du Copain est « d'exprimer publiquement la position commune des six structures (Civam44, Terroir 44, Accueil Paysan, Gab44, Confédération Paysanne, Manger Bio 44) en pointant les incohérences du projet dans la perspective d'un développement de territoire à long terme respectueux de l'Homme, de la terre nourricière et de l'Environnement, privilégiant la relocalisation de la production et l'anticipation de la transition énergétique ${ }^{13} »$. Ce n'est ni sur le papier ni sur l'internet que se manifeste principalement le Copain, mais sur le terrain, sous la double forme de l'action directe et de la réflexion collective sur le devenir de la ZAD. Les paysans du Copain se comportent un peu comme les Naturalistes en lutte, c'est-à-dire qu'ils agissent à la fois dans la perspec-

13. Communiqué juillet 2013 sur le site du GAB 44, [http://www.gab44.org/ html/index.php?id_repertoire $=88 \varepsilon$ pere $=29]$. 


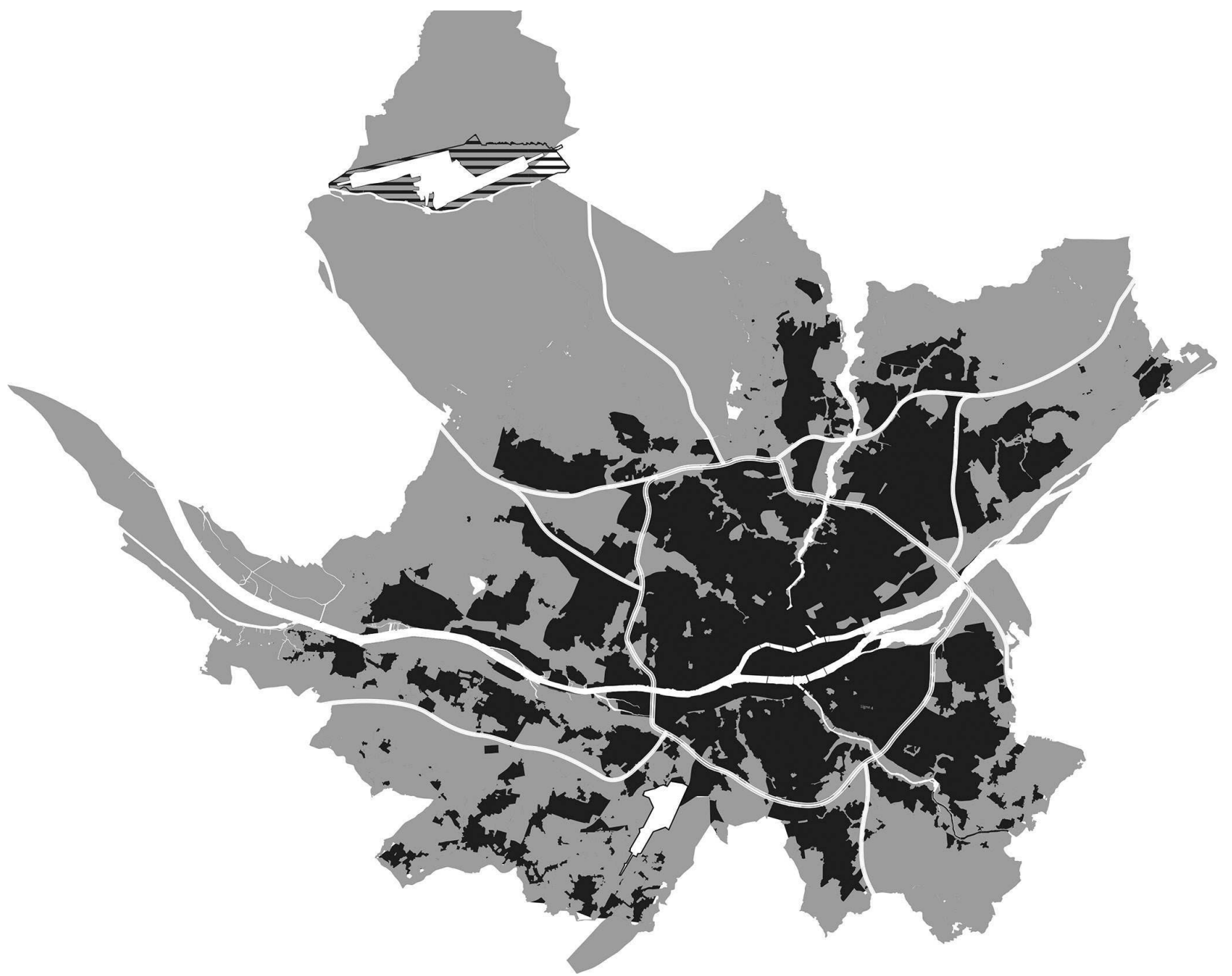

Figure 1 : Carte des ordres de grandeur métropolitains; aéroport Nantes-Atlantique, agglomération nantaise et ZAD (Mcmarco, Guide indigène de détourisme de Nantes et Saint-Nazaire, 2016, à la criée)

En noir, les zones urbanisées de la métropole; en blanc, les deux zones de controverse : au sud, l'aéroport Nantes-Atlantique, intra-métropolitain (l'aérogare est à $8 \mathrm{~km}$ du centre-ville), au nord, le périmètre du projet d'aéroport de Notre-Dame-des-Landes, hors métropole (l'aérogare est à $22 \mathrm{~km}$ du centre-ville).

Map showing metropolitan orders of magnitude: Nantes-Atlantique airport, Nantes urban area, and the ZAD (Mcmarco, Guide indigène de détourisme de Nantes et Saint-Nazaire, 2016, A La Criée).

tive d'utilité sociale qui est la leur, mais également dans une façon engagée de requalifier symboliquement et politiquement la profession agricole, c'est-àdire de se requalifier eux-mêmes. En ayant un style propre qui combine toutes les formes d'action des plus légalistes à l'action directe, voire clandestine, ils se placent au centre de la coalition. La description qu'en font des occupants dans un des nombreux reportages sonores produits par des médias alternatifs en ligne (encadré ci-dessous) me semble exprimer cela.

À la réécriture de cet article, en revisitant à nouveau souvenirs et archives, la part agricole de l'enjeu comme du mouvement me paraît bien plus forte que ce que je pensais en dire. La ferme occupée de Bellevue est devenue le principal lieu d'accueil des visiteurs de la ZAD, un lieu partagé, ouvert, habité par des occupants et visité quotidiennement par des paysans du Copain. Hors métropole, mais en lien avec des urbains, les luttes paysannes de LoireAtlantique, actualisées, incarnées ici par l'Adeca et le Copain, continuent.

L'opposition au projet d'aéroport dans la métropole nantaise semble plus fragile. Elle est notamment davantage fragmentée entre opposants institutionnels (élus divers gauche ou des listes à gauche 
"Pour moi, c'est Copain qu'a la force du mouvement en ce moment; c'est un mowvement plus ou moins uni, mais le corps, c'est le côté agricole et notamment via Copain; les paysans, comme les citoyens, y a eu l'émoi créé par l'intervention policière déjà et puis, vite, y se sont mobilisés pour que les lieux soient pas détruits, je sais pas si y faut le dire, mais en montant des barricades avec les tracteurs; le point de départ, si on peut en mettre un, c'est quand les tracteurs sont arrivés sur la ZAD [...] y avait l'envie de certains paysans de ramener des tracteurs [...] c'était le jour où y avait un rassemblement au Sabot, y avait une manif qui partait de Notre-Dame et qui devait aller an Sabot et les tracteurs sont un peu arrivés ce jour-là. [...] y a eu des paysans et des tracteurs qui se sont retrouvés à vouloir aller au Sabot et à être confrontés à des rangs de CRS et des gendarmes mobiles cons comme leurs pieds là [...] c'était pas n'importe quel lieu, y avait une histoire, un passif (agricole) [...] C'était le bordel, d'être confronté à un truc, ça fait naître un peu de solidarité et de complicité. [...] Le soir même, quand y a plus eu de flics, certains se sont organisés pour revenir et faire des barricades énormes, les premières, les plus grosses [...] Bon, moi je me disais, y va mettre trois rounds et trois arbres sur la barricade et c'est plié, ça va durer une demi-heure, en fait, non, il s'est appliqué, t'as l'impression qu'il kiffait de faire ça, il est resté des heures à organiser une barricade compliquée à bouger, t’as l'impression qu'il jouait, je sais pas ce qu'il faisait... »

Verbatim d'un extrait de l'émission « Les paysans et paysannes dans la lutte des classes » du 6 novembre 2014, Radio Canut, Lyon Transcript of an audio excerpt from the radio program "Les paysans et paysannes dans la lutte des classes" ("Men and women of the land in the class struggle"), November 6, 2014, Radio Canut, Lyon

de la gauche, élus verts des coalitions municipales Verts-PS dont c'est le principal marqueur distinctif, élus centristes, voire élus FN réclamant en même temps l'évacuation de la ZAD) et les groupes plus proches des occupants. Un ensemble de groupes variés (collectifs anti-aéroports, associations thématiques, collectifs spécialisés) évolue entre ces deux tendances. Le groupe le plus proche des occupants a organisé de très nombreuses manifestations, publié plusieurs numéros d'un journal papier intitulé «Nantes Nécropole », fait vivre le réseau anti-répression de soutien aux personnes blessées, prévenues ou incarcérées. Ce groupe a été mis en cause, avec les occupants, dans le débat autour de la violence dans la lutte, un débat interminable et certainement insoluble au regard de l'histoire sociale locale $^{14}$, et que nous ne pouvons que mentionner brièvement ici. La grande manifestation nantaise du samedi 22 février 2014 (40000 à 50000 manifestants), quasi-interdite ${ }^{15}$, puis celles, plus modestes et parfois nocturnes, liées à la mort de Rémi Fraisse le 26 octobre 2014, tué à Sivens par une grenade de

14. L'action directe du monde ouvrier et paysan y est patente (les grèves de la métallurgie de 1955, puis les grèves de la fin de la Navale, les conflits paysans avec les propriétaires fonciers des années 60 et 70 , les actions unitaires de mai 68) et elle infuse les premiers conflits environnementaux (notamment la lutte contre le projet de centrale nucléaire au Pellerin, 1976-1983, qui a connu d'importants épisodes d'action directe, à l'époque soutenus par les élus socialistes alors minoritaires et devenus depuis décisionnaires). De cette histoire sociale, reste une tradition nantaise, respectée par les grandes organisations syndicales, de ne jamais déposer de demande d'autorisation de manifestation.

15. Le parcours finalement laissé libre à la manifestation est beaucoup trop court pour en permettre l'écoulement normal. la gendarmerie mobile, ont été des moments de très grande tension entre les parties de la coalition rendant possible son éclatement - dans le contexte d'un contrôle social extrême, à la fois dans la rue et dans les médias. En 2012 et 2013, la présence policière dans la métropole et autour de la ZAD est devenue très importante. Pendant des mois, des centaines de gendarmes et de policiers stationnent dans les hôtels du département, de nombreux bâtiments sont gardés, la police développe une surveillance à caractère politique et les dispositifs de maintien de l'ordre lors des manifestations sont de plus en plus importants. Des condamnations tombent (interdictions de séjour, amendes, prison ferme). Le 22 février 2014, le dispositif policier, bloquant hermétiquement la plus grande partie du centre-ville dès le matin, apparaît véritablement impressionnant et disproportionné. Plusieurs jeunes manifestants sont blessés à l'œil par des tirs de flashball. Ils seront entendus, avec une des médecins intervenus sur la ZAD lors de l'opération César, par la Commission d'enquête sur les missions et modalités du maintien de l'ordre républicain dans un contexte de respect des libertés publiques et du droit de manifestation, le 19 mars $2015^{16}$.

Malgré ce fort contrôle social métropolitain, qui prend plus souvent la forme de la communication et de la pression, la critique s'est développée également dans l'agglomération à travers les

16. [http://www2.assemblee-nationale.fr/14/commissions-d-enquete/missionset-modalites-du-maintien-de-l-ordre-republicain-dans-un-contexte-de-respect-des-libertes-publiques-et-du-droit-de-manifestation]. 
formes de la contre-expertise. Plusieurs collectifs ou associations ont contribué à recentrer le débat sur l'aéroport Nantes-Atlantique à travers un slogan simple : "l'aéroport existe » et il est possible de le « reconstruire sur lui-même» («de l'optimiser et de le réaménager»). Le CéDpa, l'association Nexus, le Collectif des pilotes, puis en 2015, un Atelier citoyen participatif pour l'optimisation de NantesAtlantique ${ }^{17}$, coordonné par deux architectes, ainsi que la CGT ont chacun produit des analyses, des rapports et des propositions, obligeant peu à peu l'expertise officielle à évoluer. D'autre part, cinq grands projets d'ingénierie urbaine connaissent un début de mise en débat contre-expert : la rénovation de la gare de Nantes (Nexus), les transferts associés du MIN et du CHU (CFDT), le projet immobilier du marché de la Petite-Hollande (associations), la nouvelle ZAC Doulon-Gohards (un collectif de jardiniers). Enfin, dans plusieurs communes très à gauche, notamment les deux grandes communes riveraines de l'actuel aéroport, des listes situées à gauche de la gauche, clairement opposées au projet de Notre-Dame-des-Landes, ont mis en difficulté les listes Verts/PS (elles-mêmes partagées sur ce projet) : à Rezé, la liste PS est mise en ballottage historique (premier second tour depuis 1959), la liste Rezé à Gauche Toute réalise 16,08 \% et la liste d'union Écologistes/PS du premier tour, 47,39\%; à Bouguenais, la liste Bouguenais Agir Solidaire réalise un score de $27,13 \%$ et la liste Verts ${ }^{18} / \mathrm{PS}$ (fusionnée au second tour) $37,41 \%$, la liste de droite ratant de 145 voix l'élection dans un bastion de la gauche (l'un des totaux « voix de gauche » les plus élevés des municipales 2014 en France). Cette complexité nantaise révèle à la fois l'efficacité de la politique métropolitaine, mais également le développement de pratiques et de discours critiques de la métropolisation depuis l'intérieur même de la métropole.

\section{«ZAD PARTOUT » ou la mise en réseau à l'échelle nationale et européenne}

Les conflits socio-environnementaux ne sont pas une nouveauté (Dumont, 1973; Pessis et al., 2013). Les historiens de l'environnement montrent qu'ils

17. [http://www.ateliercitoyen.org/]

18. Eux-mêmes opposés au projet de nouvel aéroport, alliance qui va entraîner une inflexion de la position de la maire, demandant à son tour que le réaménagement de Nante-Atlantique soit étudié par l'expertise officielle. existent dès les premières pollutions de l'ère industrielle et que la création de la législation environnementale peut même être interprétée comme une manière de protéger les pollueurs de leurs voisins en colère (Bonneuil et Fressoz, 2013; Fureix et Jarrige, 2015) et plus récemment ses propres activités d'État aménageur et pollueur (Ost, 2003). La notion de Grand Projet Inutile Imposé (Camille, 2015) apparaît dans la Charte d'Hendaye anti-Lgv de $2010^{19}$, signée par une trentaine d'associations françaises, italiennes et espagnoles. La Charte réclame « la régénération, l'entretien et l'optimisation des voies existantes [...] la décroissance des transports, liée à une transformation profonde du modèle économique et social, en faisant notamment de la proximité et de la relocalisation de l'économie une priorité et la restitution en dernier recours de la capacité de décision aux populations directement concernées, fondement d'une authentique démocratie et autonomie locale face à un modèle de développement imposé. » Cette Charte sera suivie de cinq forums contre les Gpii - Val di Susa (Italie) août 2011, No TAV (ligne Lyon-Turin) ; Nddl, juillet 2012, aéroport Vinci; Stuttgart, juillet 2013, «Stuttgart $21 »$; Forum mondial de Tunis (et Charte de Tunis ${ }^{20}$ internationalisant la liste des signataires), mars 2013; Rosia Montana (Roumanie), mai 2014, mine d'or Gabriel Resources. À partir de 2013, des ZAD apparaissent un peu partout en France. Elles connaissent des devenirs très divers. La destruction par la police des deux ZAD emblématiques de Décines (Grand stade de Lyon/ OL-Land; Binctin, 2014) et de Sivens (projet de barrage du Testet; Collectif sans retenue, 2014) dans des contextes très différents (la deuxième agglomération française, la France des faibles densités) montre que le type de projet, son échelle, les contextes géohistoriques locaux sont déterminants (taille et qualité de la coalition opposante, enjeux réels et positions de pouvoir des porteurs de projet et de leurs alliés) au sein même de l'actualité et des rapports de force politiques nationaux. Des ZAD apparaissent à mesure que d'autres disparaissent, parce qu'elles réussissent à bloquer le processus d'un grand projet en créant les conditions d'un habiter spécifique, d'une large mise en réseau et d'une bonne médiatisation de la lutte, ainsi que d'un activisme judiciaire efficace

19. [http://lgpe.fr/Charte-d-Hendaye-Declaration].

20. [http://cadtm.org/CHARTE-de-TUNIS-adoptee-au-FSM-de]. 
(Center Parc de Roybon; Mora, 2011). Ce mouvement des ZAD apparaît fortement stigmatisé par les élus décisionnaires ou cautionnaires. Le député socialiste de Rezé-Bouguenais (rencontre, 2014) dit ainsi ne pas bien connaître le dossier du transfert de l'aéroport situé dans sa circonscription, faire confiance aux experts et aux élus en charge du dossier. Il ne souhaite pas non plus se rendre sur la ZAD ${ }^{21}$, même accompagné de militants du Sud-Loire : sa crainte, commune et rapportée ailleurs (Subra, 2003) est qu'avec les ZAD, « on ne puisse plus faire d'aménagement en France ». Nous voyons dans cette posture une position politique qui réfute la factualité des dossiers et l'habiter des lieux au profit d'intérêts jugés supérieurs, ici nommés abstraitement l'aménagement. C'est donc bien dans le cadre général d'une critique longue de la « religion du progrès » (Michéa, 2011), de la transition socio-écologique et de la réalité ou non de son existence qu'il faut replacer tous ces conflits socio-environnementaux. Certes, cet engagement ne semble concerner que peu d'individus au regard de la population générale, toutefois ce caractère minoritaire n'est pas original et la capacité de coalitions d'opposants consistantes, diverses et expérimentées, à créer ou modifier les représentations majoritaires est réelle - ce qu'un sondage de février 2014 semble montrer ${ }^{22}$. L'usage massif de la police comme mode d'extinction des ZAD (surveillance, harcèlement, expulsion, destruction) montre que le pur rapport de force demeure le paramètre caché du moment participatif aux dépens de la délibération et de la médiation. C'est aussi l'habiter en conscience d'habiter que la police défait.

Avec sa géographie en permanence remaniée par les effets de son statut de haut lieu, la ZAD est une coproduction entre habitants, occupants et visiteurs. Le haut lieu n'est pas sans effet dans les campagnes et la métropole voisines. Il inspire aussi une dynamique de passage à l'acte plus distante, en faisant

21. Dont il propose une lecture extrêmement déficitaire.

22. « $56 \%$ de Français se disent opposés au projet d'aéroport de NotreDame-des-Landes, $24 \%$ étant favorables au projet et $20 \%$ ne se prononcent pas, selon un sondage Ifop publié samedi. Le sondage a été réalisé pour le compte d'Agir pour l'environnement, d'Attac et de l'Acipa, association d'opposants au projet. Il a consisté en une seule question : "En considérant les incidences économiques, écologiques et climatiques d'un tel projet, estimez-vous que la construction du nouvel aéroport NotreDames-des-Landes doit être maintenue ou non?" " [http://www.presseocean. frlactualite/notre-dame-des-landes-sondage-plus-de-la-moitie-des-francaiscontre-laeroport-22-02-2014-9] [http://www.agirpourlenvironnement.org/ sondage-notre-dame-des-landes]. du lieu éponyme un concept en accès libre, un commun du mouvement social permettant l'appropriation d'un espace (Ripoll, 2005) ou d'un territoire, à distance du haut lieu. En ce sens, la ZAD a permis la diffusion d'un habiter en conscience d'habiter en tant que ressource politique. Comme Plogoff, la ZAD est un lieu « d'apprentissage de la mobilisation sociale »(Simon, 2010).

\section{Conclusion : L'HABITER D'UN MOUVEMENT, L'HABITER EN MOUVEMENT}

L'habiter en conscience d'habiter que nous disons avoir observé sur la zone à défendre et dans le SudLoire dans le cadre de cette recherche semble constituer une critique en actes et en corps du tournant participatif entamé il y une vingtaine d'années par les pouvoirs publics. Loin de neutraliser la conflictualité (Krieg-Planque, 2010), ces dispositifs publics d'enquête ou de débat ${ }^{23}$ cadenassés (la décision est prise, le site choisi, les terrains achetés, les matériels commandés, la parole experte mensongère ou manipulatrice) l'ont ici certainement nourrie (Verchère, 2016). La communication publique saturée de mythologie aménageuse et d'images de synthèse performatives affirme le contraire de ce que l'appareil réglementaire de l'aménagement et de la démocratie participative ${ }^{24}$ annonce par ailleurs : incertitude, acceptation des limites, nécessaire réflexivité, ouverture au débat, difficulté à dire l'intérêt général, nécessité d'articuler cultures professionnelles, politiques et habitantes - et qui fait dire à certains chercheurs de manière très provocante que « le bon aménagement, c'est en fait celui qu'on a réussi à construire » (Subra, 2013). Cette contradiction nourrit largement les «systèmes sorciers " du monde contemporain (Stengers et Pignarre, 2005) qui disqualifient les savoirs non officiels et placent les individus dans des « alternatives infernales ». De fait, selon l'aphorisme qui circule dans ce «monde de l'art » (Becker, 1988) qu'est l'aménagement français, « une enquête publique qui dys-

23. [http://cpdp.debatpublic.fr/cpdp-aeroport-ndl] pour les archives du débat public.Hiver-printemps 2003 pour la commission particulière du débat public; octobre-novembre 2006 pour l'enquête préalable à la déclaration d'utilité publique; juin-août 2012 pour l'enquête publique au titre de la loi sur l'Eau.

24. Une étude sérieuse des grands dispositifs participatifs prospectifs, hors procédures d'aménagements situés, du type « Nantes 2030 » ou «Pays de la Loire 2040 », reste encore à faire. 
fonctionne, c'est quand les habitants participent». En février 2016, la décision gouvernementale soudaine d'organiser une consultation à valeur d'avis, le 26 juin suivant, dans le seul département de la Loire-Atlantique, portant sur une seule question ( Etes-vous favorable au projet de transfert de l'aéroport de Nantes-Atlantique sur la commune de NotreDame-des-Landes? ») rejoue l'instrumentalisation des dispositifs dits participatifs. Cet objet juridique fragile ignore, par le simplisme de la question posée, la doctrine actuelle de l'aménagement français ( éviter, réduire, compenser ») comme la contreexpertise issue du conflit. La consultation apparaît comme une tentative de relégitimation électorale de l'État fragilisé pour réutiliser la force sur la ZAD et en expulser les occupants (Boudic, 2016).

Nous avons tenté de décrire et d'interpréter la contestation persistante de ce projet d'aménagement par une coalition d'acteurs hétérogènes, localement et à distance. Cette contestation est devenue celle d'un modèle de développement et du développement lui-même, entendu comme l'extension du capitalisme à de nouvelles sphères (Illich, 1973; Rist, 1996; Harvey, 2011) et dont la métropolisation et la compensation constitueraient des formes nouvelles aux échelles concernées. C'est par la mise en jeu de soi dans les lieux et les dangers que cela comporte que l'acteur social échappe à sa condition de logé, d'aménagé, de décidé, de touristifié, etc. L'habiter en conscience d'habiter définit selon nous un haut degré de mobilisation de soi au sein d'un mouvement collectif et s'accompagne d'une relation aux lieux stratégique, parfois enracinée, parfois mobile. Il s'agit de «payer de sa personne ». La rencontre de deux cultures critiques, l'une libertaire et radicale, l'autre héritière de la gauche organisationnelle y semble articulée par la tradition d'action directe paysanne et le territoire lui-même (périphérique, agricole, naturel, habité, occupé) ainsi que par la généralisation d'une sensibilité environnementale détachée de la stricte écologie politique. En concluant que l'habiter en conscience d'habiter peut aussi certainement apaiser la conflictualité, nous relions ce régime d'habiter à l'émergence d'une écologie urbaine à forte valeur culturelle (Lanaspèze, 2012) qui s'empare de la question du droit à la ville (Lefebvre, 1968; Harvey, 2011) et de de l'habitabilité (Blanc, 2010), ainsi qu'à la reconnaissance nécessaire des imaginaires indivi- duel et collectif dans la reproduction de la localité (Appadurai, 2013).

\section{Bibliographie}

Appadurai A., 2013. La condition de l'homme global, Paris, Payot, $432 \mathrm{p}$,

Barbe F., 2014. La montée des îles Aran dans la bibliothèque mondiale, une glocalisation littéraire, Cybergeo, [http:// cybergeo.revues.org/26542].

Barbe F., 2008. Interstices, Rezé, À la criée, 47 p.

Barbe F., 2014. Existe-t-il une cartographie subjective, 303, $n^{\circ} 133$, p. 44-47.

Biau V., Fenker M., Macaire É., 2013. L'implication des habitants dans la fabrication de la ville. Métiers et pratiques en question, éditions de la Villette, Cahiers Ramau nº 6, Paris, $359 \mathrm{p}$.

Binctin B., 2014. Quand le foot-business fait son grand projet inutile et imposé : le cas d'OL Land, Mowvements, $\mathrm{n}^{\circ} 78$, p. 43-54.

Blanc N., 2010. L'habitabilité urbaine, in Coutard O., LÉvy J.-P. (dir.), Écologies urbaines, Paris, Economica-Anthropos, p. 169-183.

Bonnemaison J., 1986. La dernière île, Paris, Aréla et Orstom, $408 \mathrm{p}$

Boudic G., 2016. Le référendum au risque de l'instrumentalisation, Place Publique, n ${ }^{\circ}$ 57, p. 28-33.

Bourrigaud R., 1994. Le développement agricole au $19^{\circ}$ siècle en Loire-Atlantique, Nantes, Éditions du CHT, 496 p.

Berque A., 2000. Ecoumène-Introduction à l'étude des milieux humains, Paris, Belin, 272 p.

Bey H., 1997. TAZ. Zone d'autonomie temporaire, Éditions de l'Éclat, Paris, [http://www.lyber-eclat.net/lyber/taz.html].

Bonneuil C., Fressoz J.-B., 2013. L'Événement Anthropocène. La Terre, l'histoire et nous, Paris, Le Seuil, 320 p.

Bureau Indigène de LA MAIN d'Éuvre (collectif), 2016. Guide indigène de détourisme de Nantes et Saint-Nazaire, Rezé, À la criée, 170 p.

Camille, 2015. Le petit livre noir des grands projets inutiles, Neuvy-en-Champagne, Le passager clandestin, 124 p.

Cgt 44 Et Pays de la Loire, 2015, Dossier NDDL : la démarche CGT, Nantes, communiqué, 12 p, [http://www.cgtnantesmetropole.fr/attachments/article/695/AvisNDDL-valideUDCGT44_comitéPDL.pdf].

Collectif Mauvaise Troupe, 2014. Constellations, trajectoires révolutionnaires du jeune $21^{e}$ siècle, Paris, L'Éclat, $702 \mathrm{p}$.

Collectif Mauvaise Troupe, 2016. Défendre la ZAD, Paris, L'Éclat, 46 p.

Collectif sans Retenue, 2014. Sivens sans retemue, feuilles d'automne 2014, La Lenteur, 158 p.

Collectif Sudav, 2011. C'est quoi c'tarmac? Profits, mensonges et résistances, Paris, No Pasaran, 168 p.

Collectif Z, 2010. Nantes, écologie de marché, $Z$ revue itinérante de critique sociale, numéro thématique, Montreuil, $176 \mathrm{p}$. 
Collectif ZAD Partout, 2013. ZAD Partout, zone à défendre à Notre-Dame-des-Landes - textes et images de la ZAD, Montreuil, L'insomniaque, $142 \mathrm{p}$.

De Legge J., Leguen R., 1976. Dégage!... on aménage, les Sables-d'Olonne, Le Cercle-d'Or, 147 p.

De LegGe J., 2013. Les propagandes nécessaires, éloge critique de la communication locale, Paris, Le Cherche Midi, 214 p.

Desprest V., 2009. D'un dualisme bien utile, Revue d'anthropologie des connaissances, 2009/3, p. 386-405.

Dumont R., 1973. L'utopie ou la mort, Paris, Le Seuil, 189 p.

Faburel G., 2003a. Le bruit des avions, facteur de révélation et de construction de territoires, L'espace géographique, $\mathrm{n}^{\circ} 3$, Paris, Belin, p. 205-233.

Faburel G., 2003b. Les conflits liés au bruit des avions aux USA et en Europe, repenser la place des territoires locaux dans l'environnement des aéroports, Cahiers Nantais, $\mathrm{n}^{\circ}$ 60, p. 19-29.

Fassin É, Fouteau C., Guichard S., Windels A., 2014. Roms E riverains. Une politique municipale de la race, Paris, La Fabrique, 240 p.

Fureix E., Jarrige F., 2015. La modernité désenchantée, relire l'histoire du 19e siècle français, Paris, La Découverte, 389 p.

Godet L., 2012. Conserver une nature ordinaire : une aspiration commune, des motivations différentes, Cahiers Nantais, $\mathrm{n}^{\circ} 2, \mathrm{p}$ 81-85.

Harvey D., 2011 . Le capitalisme contre le droit à la ville. Néolibéralisme, urbanisation, résistances, Paris, Amsterdam, 96 p.

Illich I., 1973. La convivialité, Paris, Le Seuil, 160 p.

Kempf H., 2014. Notre-Dame-des-Landes : le soulèvement, Paris, Le Seuil, 160 p.

Krieg-Planque A., 2010. La formule « développement durable » : un opérateur de neutralisation de la conflictualité, Langage et société, Paris, 4/2010, p 5-29.

LA BoÉtie E. de, 1547. Discours de la servitude volontaire ou le Contrun, édition préfacée par Benasayag M., 2010, Neuvyen-Champagne, Le passager clandestin, 96 p.

Lacoste Y., 1976. La géographie, ça sert d'abord à faire la guerre, Paris, Maspéro, 187 p.

Lambert B., 1970. Les paysans dans la lutte des classes, Paris, Le Seuil, 187 p.

LANASPÈze B., 2012. Marseille, ville sauvage, essai d'écologie urbaine, Arles, Actes Sud, 303 p.

Lefebvre H., 1968. Le droit à la ville, Paris, Éditions Anthropos, $164 \mathrm{p}$.

Le Madec F., 1988. L'aubépine de mai : chronique d'une usine occupée, Sud-Aviation, Nantes, 1968, Nantes, CDMOT, $144 \mathrm{p}$.

Manceron V., 2015. «Avant que nature meure »... inventorier, le cas des naturalistes amateurs en Angleterre, Ethnologie française, 2015/1 (Vol. 45), p. 31-43.

Mairie de Bouguenais, 1997. Aéroport international de NantesAtlantique de Bouguenais à Notre-Dame des Landes, un transfert à haut risque, Bouguenais, Mairie de Bouguenais, $24 \mathrm{p}$.

Marie C. dit Chirot, 2011. De la confrontation sociale à l'attrait touristique, et réciproquement, EspacesTemps.net,
Lausanne, octobre, [http://www.espacestemps.net/articles/ de-la-confrontation-sociale-a-attrait-touristique].

MichéA J.-C., 2011. Le complexe d'Orphée : la Gauche, les gens ordinaires et la religion du progrès, Paris, Flammarion, $366 \mathrm{p}$.

Moнia N., 2008. L'expérience de terrain, pour une approche relationnelle dans les sciences sociales, Paris, La Découverte, $304 \mathrm{p}$.

Mora H., 2011. Chambard dans les Chambarans, s'opposer à Center Parcs et à la marchandisation du monde, Grenoble, Le monde à l'envers, $240 \mathrm{p}$.

Monetti F., 2008. Graphes, cartes et arbres. Modèles abstraits pour une autre histoire de la littérature, Paris, Les prairies ordinaires, $139 \mathrm{p}$.

Oream, 1971. Schéma d'aménagement de l'aire métropolitaine Nantes/Saint-Nazaire, Nantes, 8 volumes, 778 p.

Ost F., 2003. La nature hors la loi, l'écologie à l'éprewve du droit, Paris, La Découverte, 346 p.

Pailloux A.-L., 2015. Zone d'aménagement différé contre «zone à défendre ». Analyse d'une lutte pour l'autonomie dans/de l'espace rural, justice spatiale/spatial justice, Nanterre, $\mathrm{n}^{\circ}$ 7, [http://www.jssj.org].

Passeron J.-C., Revel J., 2005. Penser par cas, Paris, Éditions de l'EHESS, $292 \mathrm{p}$.

Pessis C., Topçu S., Bonneuil C., 2013. Une autre histoire des "Trente Glorieuses ». Modernisation, contestations et pollutions dans la France d'après-guerre, Paris, La Découverte, $320 \mathrm{p}$.

Place Publique, 2010. L'aéroport, le savoir et la croyance, Place Publique, $\mathrm{n}^{\circ} 21$, p. 2-51.

Place Publique, 2016. Notre-Dame-des-Landes, pourquoi tant de bruit?, Place Publique, n 57, mai-juin 2016.

Pruvost G., 2015. Chantiers participatifs, autogérés, collectifs : la politisation du moindre geste, Sociologie du travail, Paris, 57 (2015), p. 81-103.

Radio Canut, 2014. Sème ta ZAD à Notre-Dame-des-Landes, émission du 6 novembre 2014, « Les paysans et paysannes dans la lutte des classes », Lyon, [http://blogs.radiocanut. org/luttespaysannes/2014/11/06/seme-ta-zad-a-notre-damedes-landes].

RANCIÈRE J., 1987. Le maître ignorant; cinq leçons sur l'émancipation intellectuelle, Paris, Fayard, $234 \mathrm{p}$.

Rancière J., 2008. Le spectateur émancipé, Paris, La Fabrique éditions, $145 \mathrm{p}$.

ReICH W., 1933 (1998). La psychologie de masse du fascisme, Paris, Payot, 528 p.

Renard J., Rialland-Juin C., 2013. Le projet d'aéroport de Notre-Dame-des-Landes : les rebonds d'un aménagement conflictuel, Géoconfluences, ENS Lyon, [http://geoconfluences.ens-lyon.fr/doc/territ/FranceMut/FranceMutDoc 17. htm].

Ripoll F., 2005. S'approprier l'espace... ou contester son appropriation?, Norois, no 195, p 29-42, [http://norois.revues. org/489]; DOI : 10.4000/norois.489.

Rist G., 1996. Le développement, histoire d'une croyance occidentale, Paris, Presses de Sciences Po, 512 p.

Rosanvallon P., 2006. La contre-démocratie, la politique à l'âge de la défiance, Paris, Le Seuil, 345 p. 
Roux J, Charvolin F., Dumain A., 2009. Les « passions cognitives » ou la dimension rebelle du connaître en régime de passion, premiers résultats d'un programme en cours, Revue d'anthropologie des connaissances, n 2009/3, p. 369 à 385.

Simon G., 2010. Plogoff. L'apprentissage de la mobilisation sociale, Rennes, PUR, 408 p.

Soulé B., 2007. Observation participante ou participation observante? Usages et justifications de la notion de participation observante en sciences sociales, Recherches qualitatives, Trois Rivières (Canada), nº 27, 127-140 p.

Stengers I., Pignarre P., 2007. La sorcellerie capitaliste. Pratiques de désenvoûtement, Paris, La Découverte, 238 p.

Stock M., 2004. L'habiter comme pratique des lieux géographiques, EspacesTemps.net, Travaux, 18.12.2004, [http:// www.espacestemps.net/articles/habiter-comme-pratique-deslieux-geographiques/].

Subra P., 2003. À quoi et à qui sert le débat public?, Hérodote, $\mathrm{n}^{\circ} 110,[$ http://www.cairn.info/revue-herodote-2003-3-p-149. $h t m]$.
Subra P., 2006. Ce que le débat public nous dit du territoire et de son aménagement, revue Géocarrefour, nº 81, [http:// geocarrefour.revues.org/1659].

Subra P., 2007. Géopolitique de l'aménagement du territoire, Paris, Armand Colin, 327 p.

Subra P., 2013. Intervention au café géo de Nantes avec Jean Renard, 27 mars 2013.

Verchère F., 2016. Notre-Dame-des-Landes : la fabrication d'un mensonge d'État, La Colle-sur-Loup, Tim Buctu éditions, [http://etatsetempiresdelalune.blogspot.fr/2016/01/un-document-evenement-sur-notre-dame.html].

Volvey A., Calbérac Y., Houssay-Holzschuch M., 2012. Terrains de je. (du) sujet (au) géographique, Annales de géographie, $\mathrm{n}^{\circ}$ 687-688, p. 441-461. 
planche IV (Frédéric BARBE - La « zone à défendre » de Notre-Dame-des-Landes ou l'habiter comme politique)

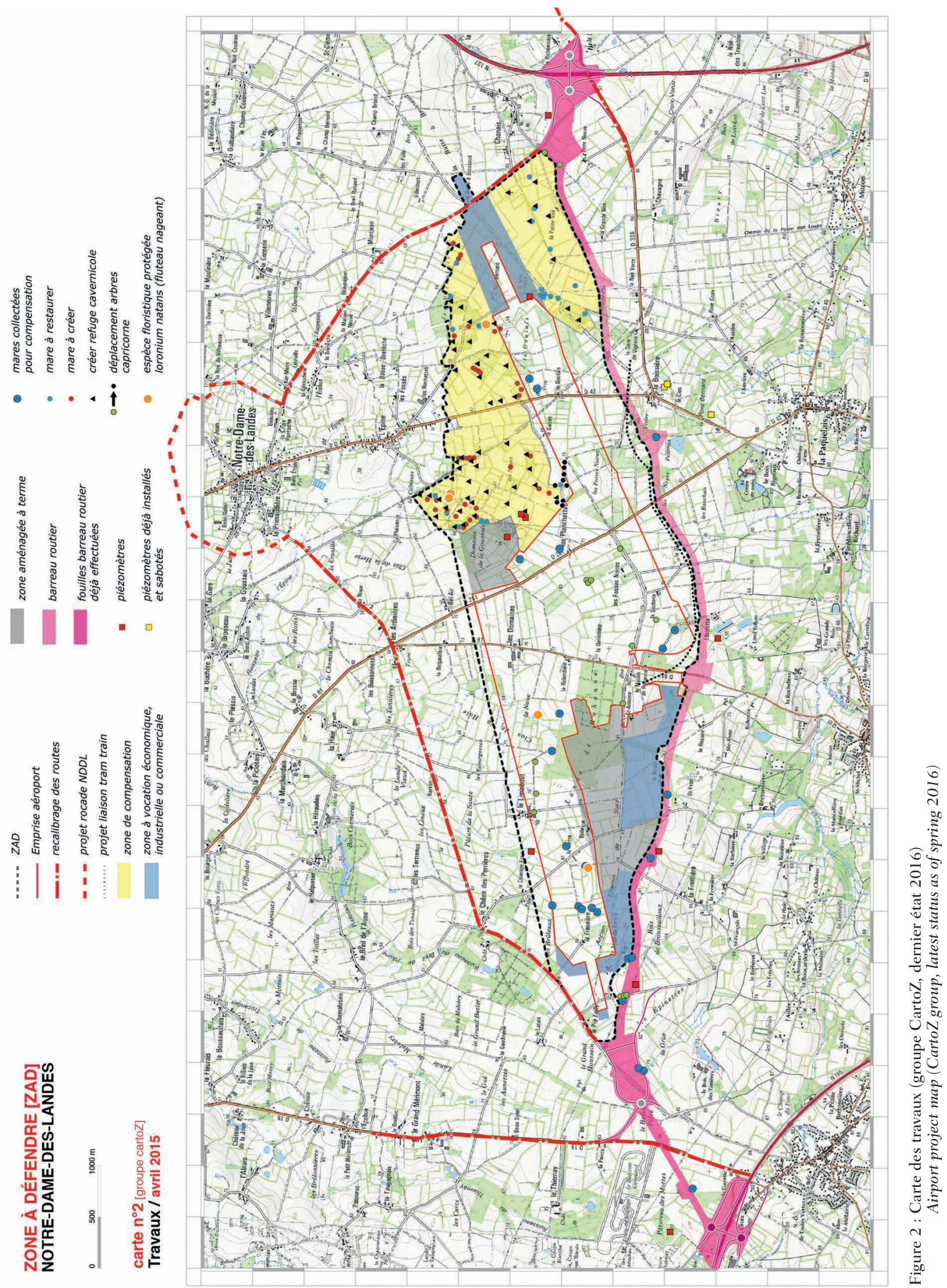


planche $\boldsymbol{V}$ (Frédéric BARBE - La «zone à défendre » de Notre-Dame-des-Landes ou l'habiter comme politique)

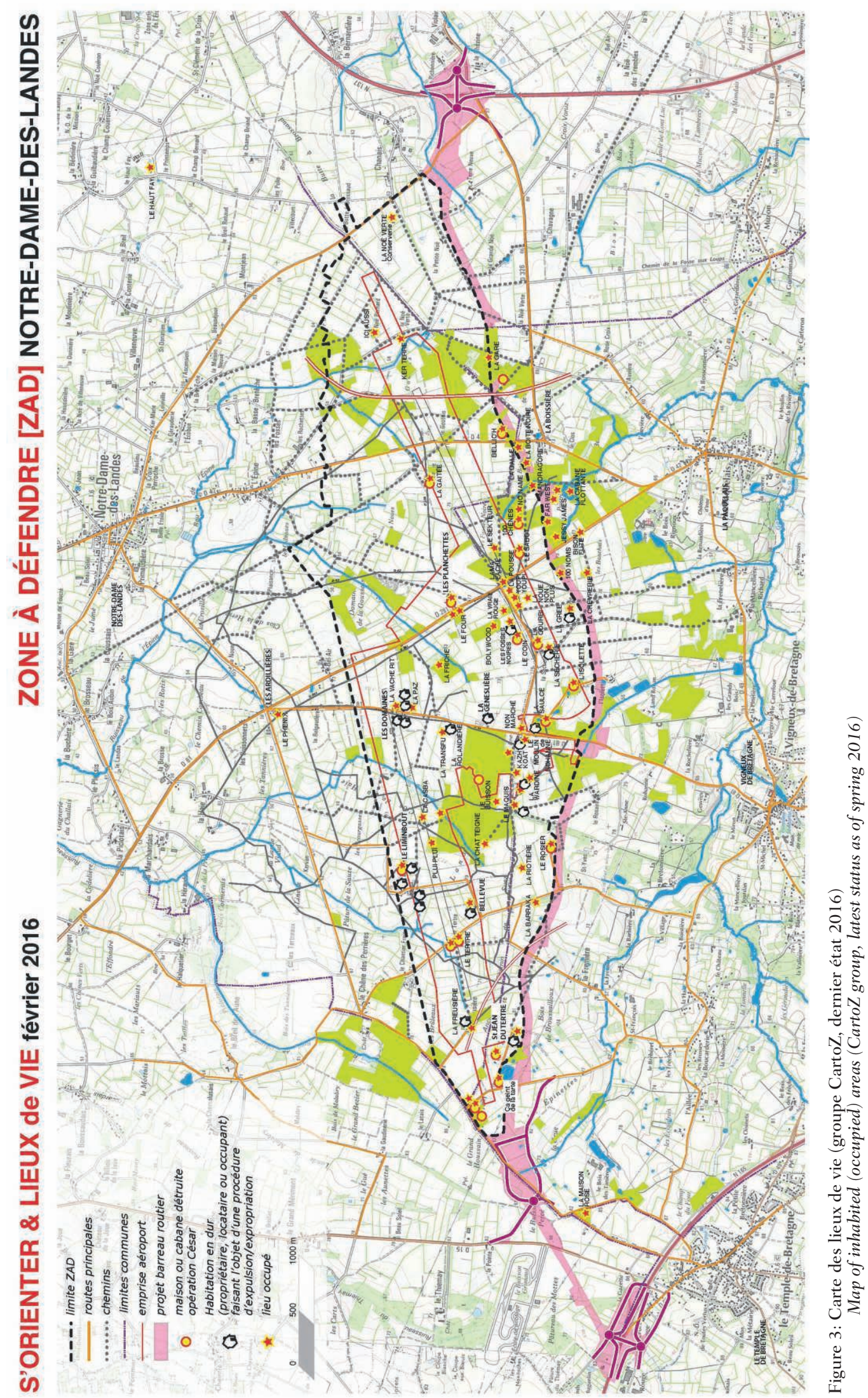


planche VI (Frédéric BARBE - La « zone à défendre » de Notre-Dame-des-Landes ou l'habiter comme politique)

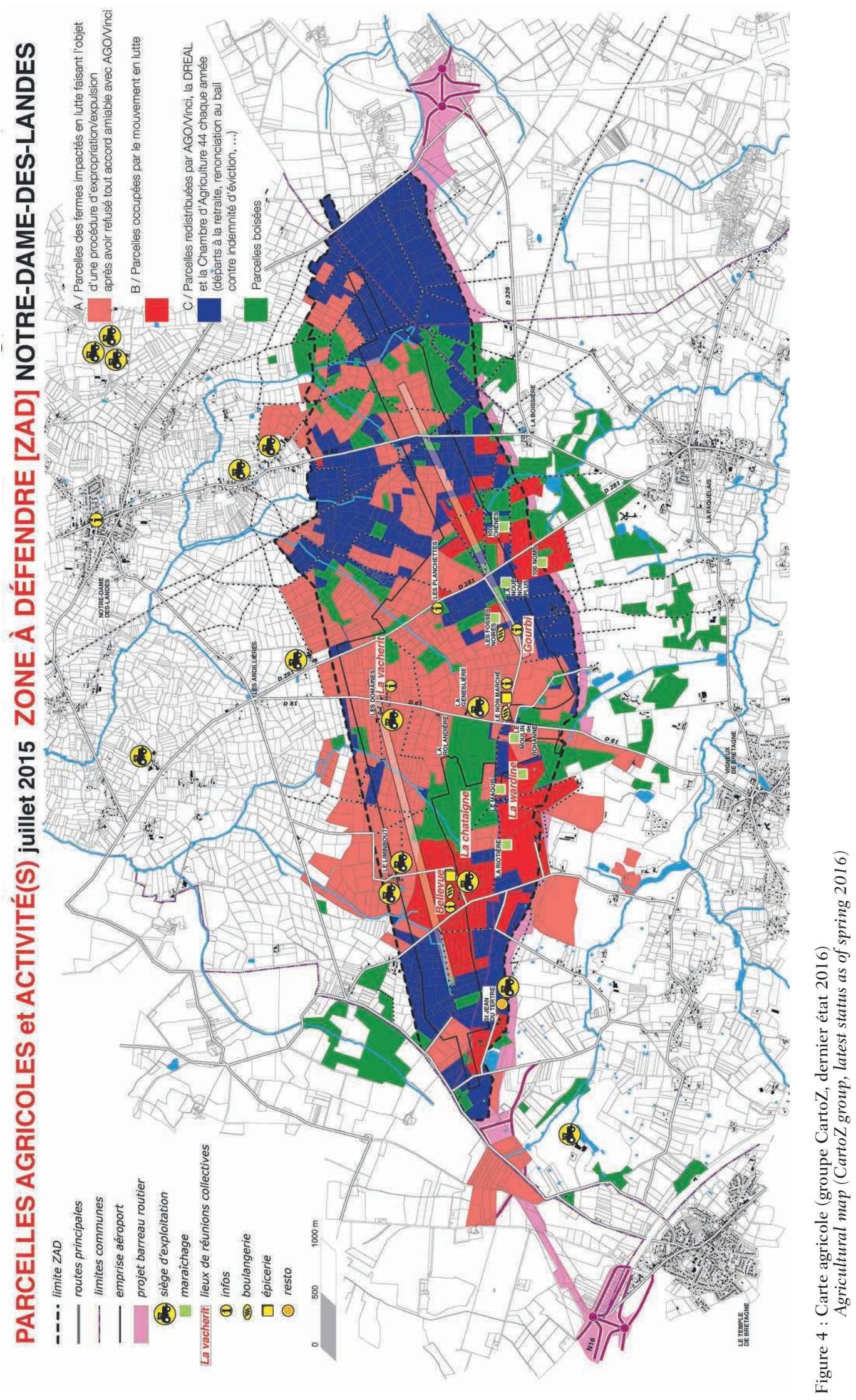


planche VII (Frédéric BARBE - La «zone à défendre » de Notre-Dame-des-Landes ou l'habiter comme politique)

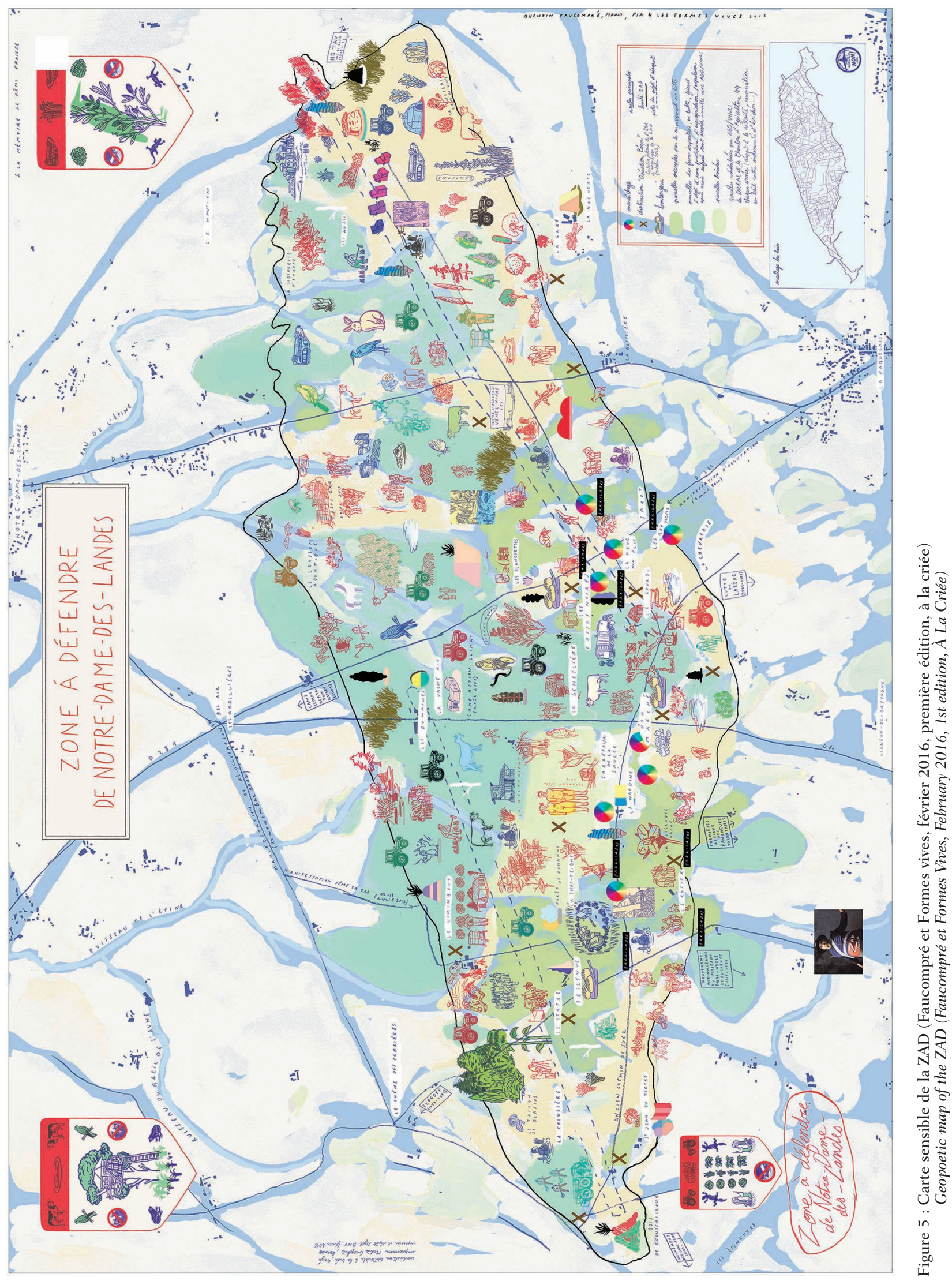

